\title{
Lateral short range ordering of step bunches in InGaAs/GaAs superlattices
}

\author{
M. Hanke ${ }^{\text {a) }}$ M. Schmidbauer, R. Köhler, and H. Kirmse \\ Institut für Physik, Humboldt-Universität zu Berlin, Newtonstrasse 15, D-12489 Berlin \\ M. Pristovsek \\ Institut für Festkörperphysik, Technische Universität Berlin, Hardenbergstrasse 36, D-10632 Berlin
}

(Received 3 October 2003; accepted 18 November 2003)

\begin{abstract}
In the present paper we report on structural investigations of fivefold $\operatorname{In}_{0.2} \mathrm{Ga}_{0.8} \mathrm{As} / \mathrm{GaAs}$ superlattices which have been grown by means of metal organic chemical vapor deposition on vicinal $\mathrm{GaAs}(001)$ substrates. Cross-sectional transmission electron micrographs exhibit an initially flat and nonfaceted grooved surface, while step bunching occurs during subsequent growth stages with an inclined vertical inheritance approximately $45^{\circ}$ off the (001) direction. A reconstructed sample cross section on the base of high resolution x-ray diffraction data qualitatively confirms the local morphology proved by transmission electron microscopy. Moreover, a line shape analysis of diffusely scattered intensity using Gauss profiles indicates a lateral short range ordering of step bunches. (C) 2004 American Institute of Physics. [DOI: 10.1063/1.1640786]
\end{abstract}

\section{INTRODUCTION}

Steps on a surface can serve as a nonartifical template for the formation of anisotropic nanostructures, such as, e.g., quantum wires $^{1}$ and two-dimensional ordered quantum dots. ${ }^{2}$ Bunches of steps-separated by extended flat terraces - tend to be much straighter than individual ones because of higher stiffness. In order to utilize those templates in real device structures one needs to control the self-assembly process. Further on, it is highly desirable to tune morphological parameters such as bunch height and separation width, which in fact can be adjusted independently by a proper choice of growth conditions. ${ }^{3}$ The strong interest in the presently investigated material combination $\mathrm{InGaAs} / \mathrm{GaAs}$ is driven by the expection of building optoelectronic devices for the 1.3 $\mu \mathrm{m}$ optical window in silica fiber, e.g., on a base of InAs quantum dots embedded within a strain-reduced InGaAs matrix. ${ }^{4}$ In addition to this technological viewpoint, step bunching has attracted fundamental interest as well. Different pathways toward step bunching have been discussed in terms of either energetics ${ }^{5-7}$ or kinetics, e.g., Refs. 8 and 9.

Recent work on molecular beam epitaxial growth of strained InGaAs on vicinal GaAs(001) wafers shows considerable uniformity and lateral order of step bunches, but only for a comparatively low highly regular step bunches indium content of $9 \% .^{10}$ We have applied metal organic chemical vapor deposition (MOCVD), which can provide even at higher strain or larger indium content.

Since scattering techniques probe extended ensembles of nanoscopic objects, they have become the method of choice for nondestructive investigations of both (i) lateral ordering within a single layer and (ii) vertically correlated interface roughness in superlattices, e.g., Refs. 11-14. Moreover, it is a sensitive technique, which can probe strain in lateral peri-

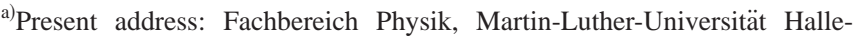
Wittenberg, Hoher Weg 8, D-06120 Halle/Saale, Germany; electronic mail: hanke@physik.uni-halle.de
}

odic structures, like those caused by a dislocation network. ${ }^{15}$

Usually ordering phenomena are discussed in the framework of two limiting ideal cases: short range ordering (SRO) and long range ordering (LRO), respectively. Whereas for LRO the correlation within domains is assumed to be perfect, ordering disappears at larger distances in SRO. ${ }^{16,17}$ Theoretical studies of the systems InAs/GaAs and $\mathrm{Si} / \mathrm{Ge}$ indicate $^{18}$ the absence of a pronounced lateral LRO of quantum dots due to the comparatively weak elastic anisotropy. We could also prove SRO for one-dimensional objects like step bunches in a vertical InGaAs/GaAs superlattice grown on a miscut surface.

\section{SAMPLE}

All the investigated samples were grown by means of MOCVD, which was performed in a double wall horizontal quartz reactor with a purged strain-reduced window for optical in situ characterization. The carrier gas was hydrogen (3 $1 / \mathrm{min}$ ) at a total pressure of $10 \mathrm{kPa}$; the $\mathrm{AsH}_{3}$ (arsine) partial pressure was $100 \mathrm{~Pa}$. The metal organic sources were trimethyl gallium (TMGa) and trimethyl indium (TMIn). A TMGa partial pressure of $0.5 \mathrm{~Pa}$ resulted in a growth rate at $873 \mathrm{~K}$ of $900 \mathrm{~nm} / \mathrm{h}$, a typical parameter for buffer growth to achieve step bunching on vicinal substrates. In the reactor there is no sample rotation implemented; thus, the thermocouple temperature was always found to be very close to the actual surface temperature. The samples were grown on vicinal $2^{\circ}[110]$ (A) and [1 $\left.\overline{1} 0\right]$ (B) oriented Te-doped $\operatorname{GaAs}(001)$ wafers. Prior to epitaxy the substrates were wet etched. Before the actual growth could start, an approximately $500 \mathrm{~nm}$ thick GaAs buffer layer was grown. At this thickness the step bunching is saturated, as also observed in the literature. ${ }^{19-21}$ On this buffer layer the vertical InGaAs/ GaAs superlattices (SLs) were grown. They consist of five periods InGaAs $(2 \mathrm{~nm})$ and GaAs $(4 \mathrm{~nm})$ with a nominal indium content of $20 \%$. 


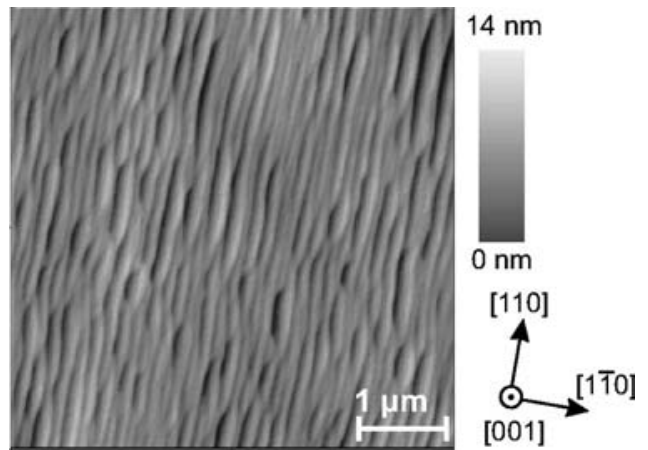

FIG. 1. The atomic force micrograph (a) shows well pronounced step bunches with an aspect ratio of approximately 10 .

We could directly observe remarkable differences in the lateral bunch aspect ratio at the topmost surface by applying atomic force microscopy. The lateral bunch aspect ratio $R$ can be defined by

$$
R=\frac{\left\langle x_{\|}\right\rangle}{\left\langle x_{\perp}\right\rangle}
$$

where $\langle\cdot\rangle$ denotes the averaged length parallel and perpendicular to the structures, respectively. Whereas the topmost bunches on a [110] (A) wafer exhibit an aspect ratio $R$ of nearly 3 (not shown here), we found a significantly higher ratio of 10 in the case of the [1 $\overline{1} 0]$ (B) wafer (see Fig. 1). Hence, we will focus on the structure grown on the [1 $\overline{1} 0]$ (B) substrate.

\section{EXPERIMENT}

The x-ray scattering experiments have been prepared at beamline BW2 at HASYLAB using monochromatic $\mathrm{x}$ rays with an energy of $8 \mathrm{keV}$ and an energy width $\Delta \lambda / \lambda$ of approximately $10^{-4}$. We made particular use of a position sensitive detector (PSD), which was placed $750 \mathrm{~mm}$ behind the sample. By using lateral slits of $1 \mathrm{~mm}$ directly behind the sample and additionally in front of the PSD we could establish a sufficiently high resolution of $4.3 \times 10^{-3} \AA^{-1}$ perpendicular to the scattering plane, whereas the much better resolution in plane amounts to $2.9 \times 10^{-4} \AA^{-1}$ parallel to the surface and $4.3 \times 10^{-4} \AA^{-1}$ perpendicular to it.

\section{RESULTS AND DISCUSSION}

Figure 2 shows the diffusely scattered intensity around the 004 lattice point for two different scattering planes. The intense streaks crossing both patterns are artifacts caused by the monochromator (marked $M$ ) and the position sensitive detector $(P)$, respectively. Figure 2(a) depicts the diffuse scattering in a plane collinear with the bunches; thus it contains the [110] direction. In the limit of perfect onedimensional steps and/or SBs there is no contribution to the corresponding scattering plane. However, the intensity distribution contains information about the vertical sample structure, which particular periodic arrangement causes intensity oscillations along the crystal truncation rod (CTR).

In contrast, Fig. 2(b) shows the distribution within a plane perpendicular to the previous one, which is defined by

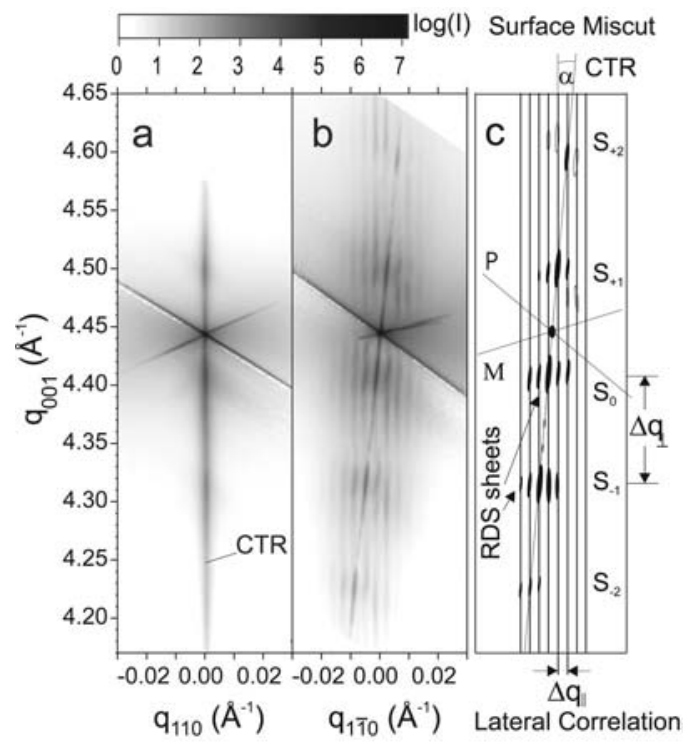

FIG. 2. Diffusely scattered intensity around the 004 reflection, where the incoming beam runs along the bunches (along the [110] direction) in (a) and perpendicular to it in (b). (c) depicts a schema of the measured distribution (b).

the $[1 \overline{1} 0]$ and $[001]$ directions. Here, different vertical and lateral features are superimposed and merge into a very complicated pattern, which parts are better discussed by means of the schematic drawing in Fig. 2(c). The monodispersely distributed step bunches and in particular their lateral periodic separation by extended (001) terraces give rise to characteristic grating truncation rods (GTRs) in the diffuse scattering up to the fifth order. Those rods are perfectly oriented along [001], indicating a lateral ordering which exactly follows the [1 $1 \overline{1} 0]$ direction. From the lateral distance $\Delta q_{\|}$of 3.76 $\times 10^{-3} \AA^{-1}$, we can estimate an averaged lateral bunch spacing $\left\langle d_{\|}\right\rangle$of $1671 \AA$. This is in good agreement with the atomic force micrograph of the topmost surface in Fig. 1. Moreover, the cross-sectional transmission electron micrograph, Fig. 3, depicts nearly the same bunch separation for

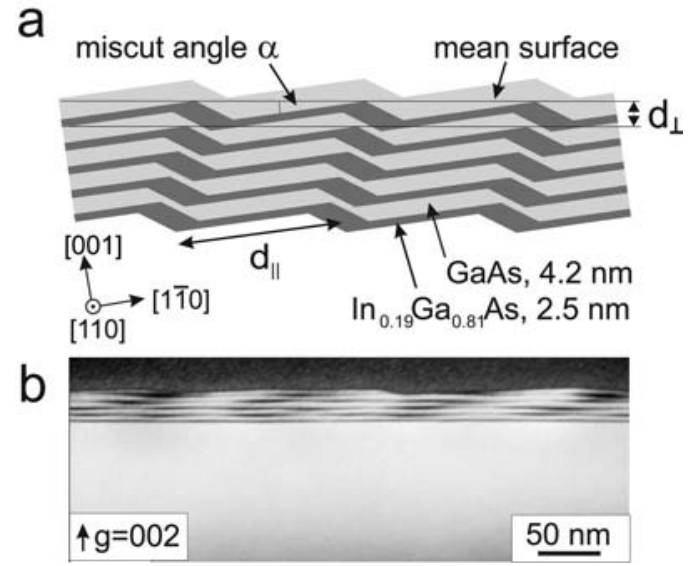

FIG. 3. A schematic cross section of the sample as derived from the lateral and vertical periodicity and location in high resolution $\mathrm{x}$-ray diffraction data, which is in excellent agreement with the cross-sectional transmission electron micrograph (b). It is worth noting that step bunching subsequently develops during growth since there is no substrate prepattern visible. 
the underlying interfaces. A very intense crystal truncation rod crosses the whole diffuse intensity pattern, at an angle of $2.3^{\circ}$ which confirms the initial substrate miscut.

Moreover, the vertical superstructure (five periods of InGaAs/GaAs) causes equidistantly spaced vertical superlattice peaks $\left(S_{-2, \ldots,+2}\right)$, whose separation $\Delta q_{\perp}$ is closely related to the SL period $d_{\mathrm{InGaAs}}+d_{\mathrm{GaAs}}$, where the distance between the zeroth order SL peak reflects the strain averaged along the entire stack. Dynamical scattering simulations (not shown here) reveal layer thicknesses of $d_{\mathrm{GaAs}}=4.2 \mathrm{~nm}$ and $d_{\mathrm{InGaAs}}=2.5 \mathrm{~nm}$ and an averaged indium concentration of $x_{\text {In }}=19 \%$. Since the lateral maxima at various SL peaks are inclined with respect to the [001] direction, the vertical inheritance is supposed to be inclined.

A detailed inspection of Fig. 2(b) indicates that the SB height has to be identical to the vertical SL period $d_{\text {InGaAs }}$ $+d_{\text {GaAs }}$ itself, since the CTR obviously crosses the lateral GTRs exactly at the maxima of subsequent SL peaks, which yields the relation

$$
\sin \alpha=\Delta q_{\|} / \Delta q_{\perp}=\left\langle d_{\perp}\right\rangle /\left\langle d_{\|}\right\rangle,
$$

where $\alpha$ denotes the miscut angle.

Taking this morphological particularity into account, a cross section through the discussed structure has been reconstructed. Figure 3(a) depicts a possible configuration considering the results by high resolution $\mathrm{x}$-ray diffraction (HRXRD). The cross-sectional transmission electron micrograph in Fig. 3(b) qualitatively confirms the proposed model.

All the micrographs give clear evidence that step bunches do not immediately emerge at the initial interface, which is free of any undulation. Therefore step bunching and its vertical inheritance are rather phenomena driven by strain relief during subsequent growth than a simple replication of a prepattern.

The existence of correlation peaks up to high orders enables one to distinguish between SRO and LRO models. Therefore we have extracted a horizontal one-dimensional intensity cut along $q_{[1 \text { [10] }}$ at $q_{z}=4.316 \AA^{-1}$ [Fig. 4(a)]. The correlation peaks are equidistantly spaced, yielding the mean lateral distance $\left\langle d_{\|}\right\rangle$. On the other hand, the experimental peak widths $\delta q$ [shown as dots in Fig. 4(b)] increase with peak order. Since the connecting parabolic fit (line) does not exactly match the experimental data, the "random walk" SRO model cannot perfectly describe the morphology. However, applying this model we estimate a rms standard deviation $\sigma=166 \AA$ of the mean lateral distance $\left\langle d_{\|}\right\rangle$.

\section{CONCLUSION}

In conclusion, we have investigated MOCVD grown InGaAs/GaAs fivefold stacks on $2^{\circ}$ [110] (A) and [1 $\left.\overline{1} 0\right]$ (B) GaAs (001) wafers by HRXRD and direct imaging techniques like transmission electron and atomic force microscopy. The observed step bunches at the topmost surface with a mean lateral distance between 150 and $200 \mathrm{~nm}$ have to be formed during subsequent growth, since the initial GaAs miscut surface does not show a faceted grooved morphology. The particular shape of diffuse $x$-ray scattering in the vicinity of the 004 reciprocal lattice point indicates a step bunch

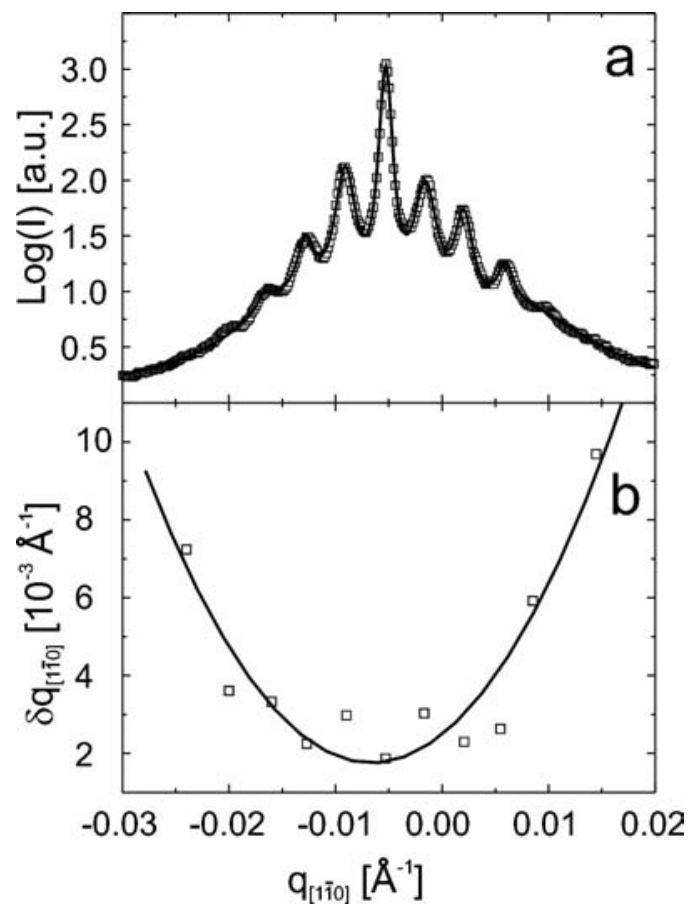

FIG. 4. (a) Experimental intensity cut (dots) along $q_{[1 \mathrm{I} 0]}$ at $q_{z}$ $=4.316 \AA^{-1}$ through the diffuse intensity pattern in Fig. 2(b) connected by a Gaussian fit. (b) plots the experimental peak widths (full widths at half maximum) (dots) as a function of $q_{[1 \overline{1} 0]}$ and a corresponding parabolic fit.

height that is nearly equal to the vertical superlattice period. Taking the experimentally estimated layer thicknesses and the actual miscut angle into account, a typical sample cross section has been reconstructed. Line shape analysis of onedimensional cuts through the first order vertical superlattice peak of the 004 reciprocal space map proves lateral short range ordering.

\section{ACKNOWLEDGMENTS}

We would like to thank W. Drube for the experimental setup at beamline BW2 at Hasylab. This project was sponsored by the German Research Society in the framework of the Sonderforschungsbereich 296.

${ }^{1}$ K. Ploog, R. Nötzel, and M. Ilg, J. Vac. Sci. Technol. B 11, 1675 (1993).

${ }^{2}$ J. Zhu, K. Brunner, and G. Abstreiter, Appl. Phys. Lett. 73, 620 (1998).

${ }^{3}$ F. Liu, J. Tersoff, and M. G. Lagally, Phys. Rev. Lett. 80, 1268 (1998).

${ }^{4}$ Y. Qiu, P. Gogna, S. Forouhar, A. Stintz, and L. Lester, Appl. Phys. Lett. 79, 3570 (2001).

${ }^{5}$ J. Tersoff, Y. H. Phang, Z. Zhang, and M. G. Lagally, Phys. Rev. Lett. 75, 2730 (1995).

${ }^{6}$ D.-J. Liu and J. Weeks, Phys. Rev. Lett. 79, 1694 (1997).

${ }^{7}$ Z. M. Wang, L. Däweritz, and K. H. Ploog, Surf. Sci. 459, L482 (2000).

${ }^{8}$ M. Krishnamurthy, M. Wassermeier, D. R. M. Williams, and P. M. Petroff, Appl. Phys. Lett. 62, 1922 (1993).

${ }^{9}$ C. Duport, P. Nozières, and J. Villain, Phys. Rev. Lett. 74, 134 (1995).

${ }^{10}$ Z. Wang, J. Shultz, and G. Salamo, Appl. Phys. Lett. 83, 1749 (2003).

${ }^{11}$ Y. H. Phang, R. Kariotis, D. E. Savage, and M. G. Lagally, J. Appl. Phys. 72, 4627 (1992).

${ }^{12}$ Z. H. Ming, A. Krol, Y. L. Soo, Y. H. Kao, J. S. Park, and K. L. Wang, Phys. Rev. B 47, 16373(1993).

${ }^{13}$ V. M. Kaganer, S. A. Stepanov, and R. Köhler, Phys. Rev. B 52, 16369 (1995).

${ }^{14}$ M. Schmidbauer, R. Opitz, T. Wiebach, and R. Khler, Phys. Rev. B 64, 
195316 (2001)

${ }^{15}$ J. Eymery, D. Buttard, F. Fournel, H. Moriceau, G. Baumbach, and D. Lübbert, Phys. Rev. B 65, 165337 (2002).

${ }^{16}$ J. Stangl et al., J. Vac. Sci. Technol. B 18, 2187 (2000).

${ }^{17}$ I. Kegel, T. H. Metzger, J. Peisl, P. Schittenhelm, and G. Abstreiter, Appl. Phys. Lett. 74, 2978 (1999).
${ }^{18}$ V. Holý, G. Springholz, M. Pinczolits, and G. Bauer, Phys. Rev. Lett. 83, 356 (1999).

${ }^{19}$ T. Fukui and H. Saito, Jpn. J. Appl. Phys., Part 2 29, L483 (1990).

${ }^{20}$ M. Kasu and T. Fukui, Jpn. J. Appl. Phys., Part 2 31, 864 (1992).

${ }^{21}$ J. Ishizaki, K. Ohkuri, and T. Fukui, Jpn. J. Appl. Phys., Part 1 35, 1280 (1996). 